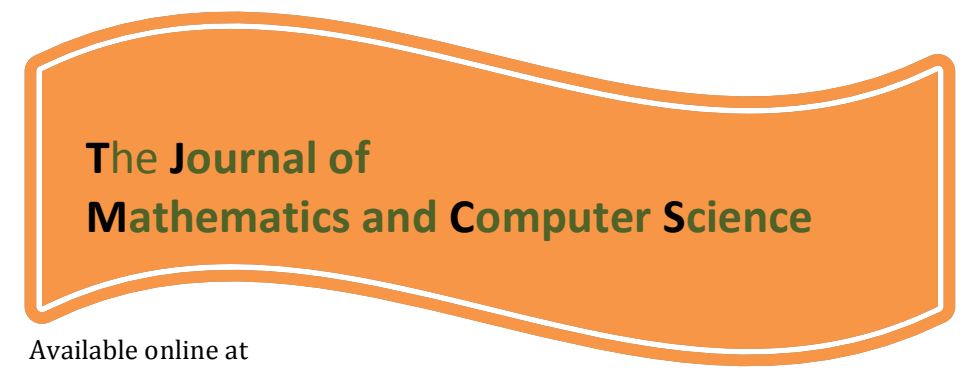

http://www.TJMCS.com

The Journal of Mathematics and Computer Science Vol.3 No.2 (2011) 212 - 224

\title{
Modification of the Homotopy Perturbation Method for Numerical Solution of Nonlinear Wave and System of Nonlinear Wave Equations
}

\author{
B. Ghazanfari ${ }^{\mathrm{a}}$, A.G. Ghazanfari a , M. Fuladvand ${ }^{\mathrm{a}}$ \\ a Department of Mathematics, Faculty of Science, \\ Lorestan University , 68137-17133 \\ Khorramabad, Iran \\ E-mail: bahman_ghazanfari@yahoo.com \\ E-mail: ghazanfari.amir@gmail.com \\ E-mail: minafuladvand@yahoo.com
}

Received: May 2011, Revised: June 2011

Online Publication: December 2011

\begin{abstract}
In this paper, the modification of the homotopy perturbation method (MHPM) Zaid M. Odibat (Appl. Math. Comput. (2007)) is extended to derive approximate solutions of the nonlinear coupled wave equations. This work will present a numerical comparison between the modification and the homotopy perturbation method (HPM). In order to show the ability and reliability of the method some examples are provided. The results reveal that the method is very effective and simple. The modified method accelerates the rapid convergence of the series solution and reduces the size of work.
\end{abstract}

Key Words: Homotopy purturbation method; Nonlinear differential equations; Modified homotopy perturbation method

${ }^{*}$ Corresponding author: Tel/Fax: +98 6612218013 


\section{Introduction}

To find the explicit solutions of nonlinear differential equations, many powerful methods have been used. The homotopy perturbation method (HPM) [1] was first proposed by He in 1998. The applications of the HPM [1-17] have appeared in a lot of researches, especially during recent years, which show that the method is a powerful technique for studying the numerical solutions.

Motivated by the above works [1-17], we would like to extend the application of the MHPM [11] to the nonlinear wave and system of nonlinear wave equations. This modification demonstrates a rapid convergence of the series solution if compared with standard HPM and, therefore, it has been shown to be computationally efficient in several examples in applied fields. In addition, the modified algorithm may give the exact solution for nonlinear equations by using one iteration only. The obtained results suggest that this method introduces a powerful improvement for solving systems of nonlinear problems.

In this subsections, we will present a review of the HPM and the MHPM [11].

\subsection{Homotopy perturbation method}

The Principles of the HPM and its applicability for various kinds of differential equations are given in many records [1-10]. Consider the nonlinear differential equations

$$
L(u)+N(u)=f(r), \quad r \in \Omega,
$$

with boundary conditions

$$
\mathrm{B}\left(\mathrm{u}, \frac{\partial u}{\partial \boldsymbol{n}}\right)=0, \quad \mathrm{r} \in \Gamma,
$$

The He's homotopy perturbation technique defines the homotopy

$v(r, p): \Omega \times[0,1] \rightarrow \mathrm{R}$, which satisfies

$$
H(v, p)=(1-p)\left[L(v)-L\left(u_{0}\right)+p[L(v)+N(v)-f(r)]=0,\right.
$$

or

$$
H(v, p)=L(v)-L\left(u_{0}\right)+p L\left(u_{0}\right)+p[N(v)-f(r)]=0,
$$

where $r \in \Omega$ and $p \in[0,1]$ is an embedding parameter, $u_{0}$ is an initial approximation which satisfies the boundary conditions and $\boldsymbol{n}$ is the unit outward normal of $\Omega$. Obviously, from Eqs. (2) and (3), we have 


$$
\begin{aligned}
& H(v, p)=L(v)-L\left(u_{0}\right)=0, \\
& H(v, 1)=L(v)+N(v)-f(r)=0,
\end{aligned}
$$

The changing process of $p$ from zero to unity is just that of $v(r, p)$ from $u_{0}$ to $u(r)$. In topology, this so-called deformation, $L(v)-L\left(u_{0}\right)$ and $L(v)+N(v)-f(r)$ are homotopic. The basic assumption is that the solution of (2) and (3) can be expressed as a power series in $p$ :

$$
v=v_{0}+p v_{1}+p^{2} v_{2}+\cdots .
$$

The approximate solution of (1), therefore, can be readily obtained :

$$
u=\lim _{p \rightarrow 1} v=v_{0}+v_{1}+v_{2}+\cdots
$$

The series (7) is convergent for most cases, and the rate of convergence depends on $A(u)=L(u)+N(u)[17]$.

\subsection{The modified form of the HPM}

The modified form of the HPM can be established based on the assumption that the function $f(r)$ can be divided into two parts, namely $f_{0}$ and $f_{1}$,

$$
f(r)=f_{0}(r)+f_{1}(r)
$$

According to this assumption, $f(r)=f_{0}(r)+f_{1}(r)$, we can construct the homotopy $v(r, p): \Omega \times[0,1] \rightarrow \mathrm{R}$, which satisfies

$$
H(v, p)=(1-p)\left[L(v)-L\left(u_{0}\right)+p\left[L(v)+N(v)-f_{1}(r)\right]=f_{0}(r)\right.
$$

or

$$
H(v, p)=L(v)-L\left(u_{0}\right)+p L\left(u_{0}\right)+p\left[N(v)-f_{1}(r)\right]=f_{0}(r),
$$

Here, a slight variation was proposed only on the components $u_{0}$ and $u_{1}$. The suggestion was that only the part $f_{0}$ be assigned to the zeroth component $u_{0}$, whereas the remaining part $f_{1}$ be combined with the component $u_{1}$. If we set $f_{1}(r)=f(r)$ and $f_{0}(r)=0$, then the homotopy (9) or (10) reduces to the homotopy (2) or (3), respectively. However, the success of the method depends on the proper selection of the functions $f_{0}$ and $f_{1}$. 


\section{Applications}

Example 1. Consider the following nonlinear wave equation, with the initial conditions :

$$
\left\{\begin{array}{l}
\frac{\partial^{2} u}{\partial x^{2}}-u \frac{\partial^{2} u}{\partial t^{2}}=1-\frac{x^{2}+t^{2}}{2}, \quad 0 \leq x, t \leq 1 \\
u(0, t)=\frac{t^{2}}{2}, \quad \frac{\partial}{\partial x} u(0, t)=0
\end{array}\right.
$$

Exact solution of this equation is

$$
u(x, t)=\frac{x^{2}+t^{2}}{2}
$$

The standard HPM(SHPM). By using Eq. (3), a homotopy can be readily constructed as follows :

$$
\frac{\partial^{2} u}{\partial x^{2}}-\left(1-\frac{x^{2}+t^{2}}{2}\right)-p u \frac{\partial^{2} u}{\partial t^{2}}=0
$$

Substituting (6) into (12), and equating the terms with identical powers of $p$, we have

$$
\begin{aligned}
& p^{0}: \frac{\partial^{2} v_{0}}{\partial x^{2}}=\left(1-\frac{x^{2}+t^{2}}{2}\right) \quad v_{0}(x, t)=\frac{t^{2}}{2}+\frac{x^{2}}{2}-\frac{x^{4}}{24}-\frac{x^{2} t^{2}}{4}, \\
& p^{1}: \frac{\partial^{2} v_{1}}{\partial x^{2}}=v_{0} \frac{\partial^{2} v_{0}}{\partial t^{2}} \quad v_{1}(x, t)=\frac{x^{4}}{24}-\frac{7 x^{6}}{720}+\frac{x^{8}}{2688}-\frac{x^{2} t^{2}}{4}-\frac{x^{4} t^{2}}{24}+\frac{x^{6} t^{2}}{240}, \\
& p^{2}: \quad \frac{\partial^{2} v_{2}}{\partial x^{2}}=v_{0} \frac{\partial^{2} v_{1}}{\partial t^{2}}+v_{1} \frac{\partial^{2} v_{0}}{\partial t^{2}} \\
& v_{2}(x, t)=\frac{7 x^{6}}{720}+\frac{x^{4} t^{2}}{24}-\frac{327 x^{8}}{241920}+\frac{x^{8} t^{2}}{1120}-\frac{x^{10} t^{2}}{21600}+\frac{1038 x^{10}}{3628800}-\frac{x^{12}}{107520},
\end{aligned}
$$

In this manner, the remaining components of the homotopy perturbation solution can be obtained. The approximate solution of Eq. (11) obtained by means of 3-terms, is as follow :

$$
u=v_{0}+v_{1}+v_{2} .
$$


Figs. 1 and 2 show the exact and approximate solutions by SHPM.

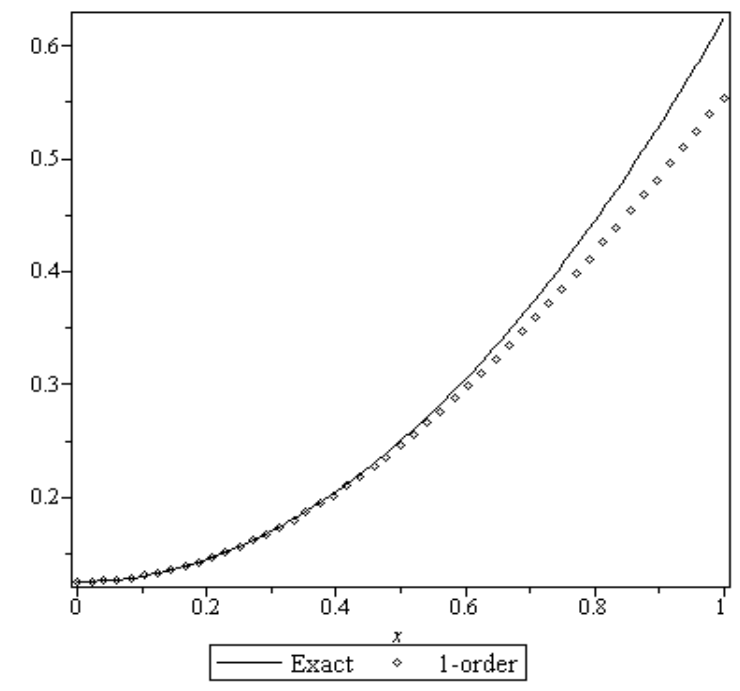

Fig. 1: Exact and 1-order of approximate solution by SHPM at $t=0: 5$.

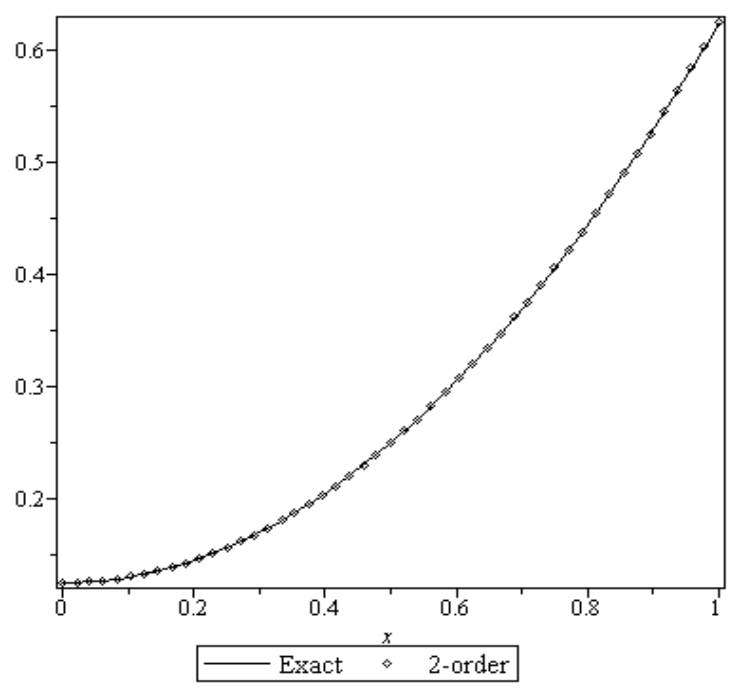

Fig. 2: Exact and 2-order of approximate solution by SHPM at $t=0: 5$.

The modified HPM. In view of the homotopy (10), we construct the following homotopy

$\frac{\partial^{2} u}{\partial x^{2}}-1+p\left(-u \frac{\partial^{2} u}{\partial t^{2}}+\frac{x^{2}+t^{2}}{2}\right)=0$

or

$\frac{\partial^{2} u}{\partial x^{2}}=1-p\left(\frac{x^{2}+t^{2}}{2}-u \frac{\partial^{2} u}{\partial t^{2}}\right)$

Substituting (6) into (13), and equating the terms with identical powers of $p$, we have

$$
\begin{aligned}
& p^{0}: \frac{\partial^{2} v_{0}}{\partial x^{2}}=1 \quad v_{0}(x, t)=\frac{x^{2}+t^{2}}{2}, \\
& p^{1} \quad: \frac{\partial^{2} v_{1}}{\partial x^{2}}=0 \quad \square v_{1}(x, t)=0 . \quad \ldots
\end{aligned}
$$

Consequently, solving the above equations, the first few components of the homotopy perturbation solution for Eq. (11) are derived as follows:

$$
v_{0}(x, t)=\frac{x^{2}+t^{2}}{2}, \quad v_{k}=0, \quad k \geq 1
$$

The exact solution $u(x, t)=\frac{x^{2}+t^{2}}{2}$ follows immediately. The success of obtaining the exact solution by using two iterations is a result of the proper selection of $f_{0}(r)$ and $f_{1}(r)$. 
Example 2. Consider the following nonlinear differential equation, with the initial conditions :

$$
\left\{\begin{array}{l}
\frac{\partial^{2} u}{\partial x^{2}}-u^{2} \frac{\partial^{2} u}{\partial t^{2}}=2\left(1-\left(x^{2}+t^{2}\right)^{2}\right), \quad 0 \leq x, t \leq 1, \\
u(0, t)=t^{2}, \quad \frac{\partial}{\partial x} u(0, t)=0 .
\end{array}\right.
$$

The exact solution of this equation is

$$
u(x, t)=x^{2}+t^{2}
$$

The standard HPM. By using Eq. (3), a homotopy can be readily constructed as follows:

$$
\frac{\partial^{2} u}{\partial x^{2}}-\left(2-2\left(x^{2}+t^{2}\right)^{2}\right)+p u^{2} \frac{\partial^{2} u}{\partial t^{2}}=0
$$

Substituting (6) into (15) and equating the terms with identical powers of $p$, we have

$$
\begin{aligned}
p^{0}: \frac{\partial^{2} v_{0}}{\partial x^{2}}=2 & -2\left(x^{2}+t^{2}\right)^{2} \\
v_{0}(x, t) & =t^{2}+x^{2}-\frac{1}{15} x^{6}-\frac{1}{3} x^{4} t^{2}-x^{2} t^{4} \\
p^{1}: \frac{\partial^{2} v_{1}}{\partial x^{2}}= & v_{0}^{2} \frac{\partial^{2} v_{0}}{\partial t^{2}} \\
v_{1}(x, t)= & \frac{1}{3} t^{2} x^{4}+t^{4} x^{2}+t^{2}+\frac{1}{15} x^{6}-\frac{1}{30} t^{12} x^{6}-\frac{7}{675} x^{10}-\frac{6}{35} t^{2} x^{8}-\frac{49}{90} t^{4} x^{6} \\
& -\frac{5}{6} t^{6} x^{4}-\frac{1}{2} t^{8} x^{2}+\frac{5}{297} x^{12} t^{2}+\frac{206}{2025} t^{4} x^{10}+\frac{3}{10} t^{6} x^{8} \\
& +\frac{19}{45} t^{8} x^{6}-\frac{151}{40950} t^{4} x^{14}+\frac{1}{6} x^{4} t^{10}-\frac{1}{34425} x^{18}-\frac{1}{12} t^{10} x^{8}-\frac{53}{2970} t^{6} x^{12} \\
& +\frac{31}{20475} x^{14}-\frac{221}{4050} t^{8} x^{10}-\frac{1}{2250} t^{2} x^{16} ., \ldots
\end{aligned}
$$

In this manner, the remaning components of the homotopy perturbation solution can be obtained. This term clearly indicate that homotopy perturbation method does not obtain efficient result to exact solution. Figs. 3 and 4 show the exact and approximate solutions by SHPM. 

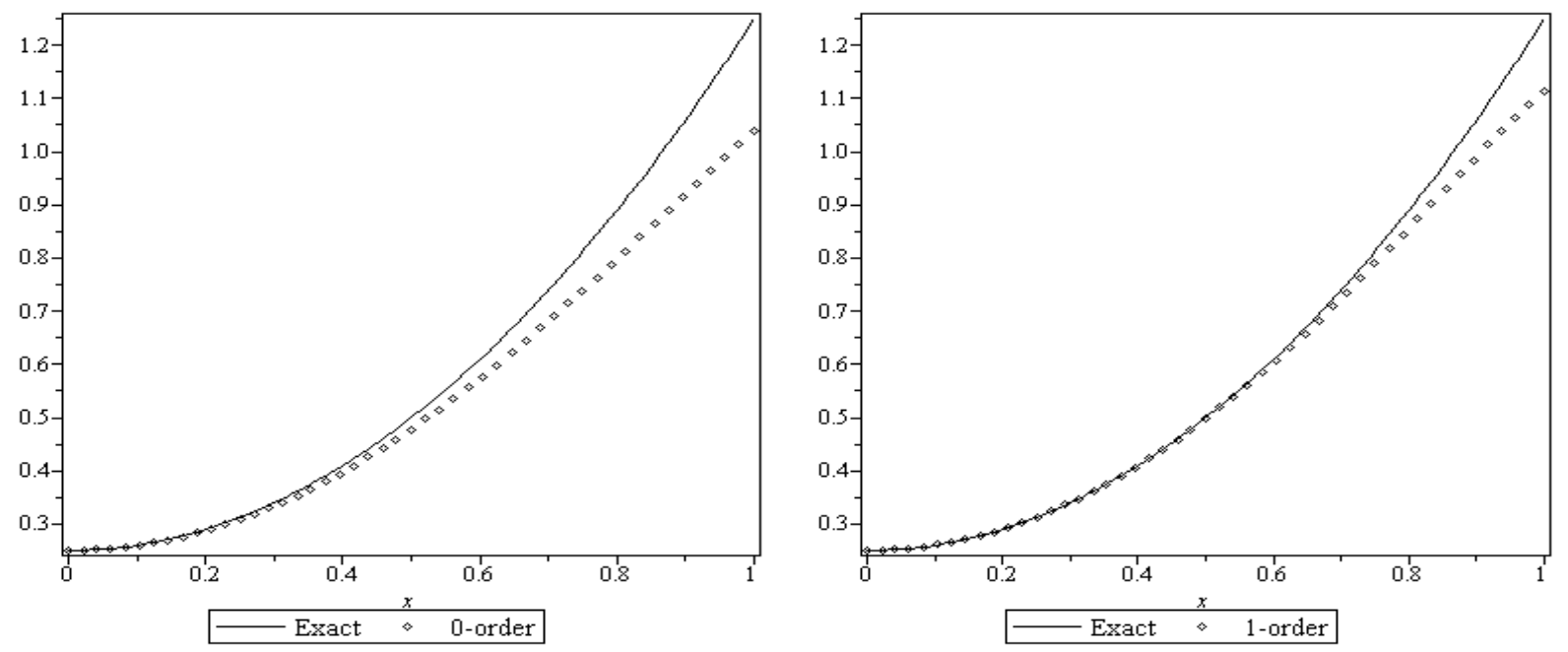

Fig. 3: Exact and 0-order of approximate solution by SHPM at $t=0: 5$. Fig. 4: Exact and 1-order of approximate solution by SHPM at $t=0: 5$.

The modified HPM. In view of the homotopy (10), we construct the following homotopy

$$
\frac{\partial^{2} u}{\partial x^{2}}=2-p\left(-u^{2} \frac{\partial^{2} u}{\partial t^{2}}+2\left(x^{2}+t^{2}\right)^{2}\right)=0 .
$$

Substituting (6) into (16) and equating the terms with identical powers of $p$, we have

$$
\begin{aligned}
& p^{0}: \frac{\partial^{2} v_{0}}{\partial x^{2}}=2 \quad \nabla_{0}(x, t)=x^{2}+t^{2}, \\
& p^{1}: \frac{\partial^{2} v_{1}}{\partial x^{2}}=0 \quad{ }_{v_{1}}(x, t)=0 . \ldots
\end{aligned}
$$

Consequently, solving the above equations, the first few components of the homotopy perturbation solution for Eq. (14) are derived as follows:

$$
v_{0}(x, t)=x^{2}+t^{2}, \quad v_{k}=0, \quad k \geq 1
$$

The exact solution $u(x, t)=x^{2}+t^{2}$ follows immediately.

Example 3. Consider the following nonlinear diferential equation, with the initial conditions :

$$
\left\{\begin{array}{l}
\frac{\partial^{2} u}{\partial x^{2}}+u \frac{\partial^{2} u}{\partial t^{2}}=4 e^{-t+2 x}+e^{-2 t+4 x} \\
u(0, t)=e^{-t}, \quad \frac{\partial}{\partial x} u(0, t)=2 e^{-t}
\end{array}\right.
$$

The exact solution of this equation is

$$
u(x, t)=e^{-t+2 x} .
$$


The standard HPM. By using Eq. (3), a homotopy can be readily constructed as follows:

$$
\frac{\partial^{2} u}{\partial x^{2}}-\left(4 e^{-t+2 x}+e^{-2 t+4 x}\right)+p u \frac{\partial^{2} u}{\partial t^{2}}=0
$$

Substituting (6) into (18) and equating the terms with identical powers of $p$, we have

$$
\begin{aligned}
p^{0}: & \frac{\partial^{2} v_{0}}{\partial x^{2}}=4 e^{-t+2 x}+e^{-2 t+4 x} \\
\quad v_{0}(x, t) & =e^{-t+2 x}+\frac{1}{16} e^{-2 t+4 x}-\frac{1}{4} x e^{-2 t}-\frac{1}{16} e^{-2 t} \\
p^{1}: \frac{\partial^{2} v_{1}}{\partial x^{2}}= & -v_{0}^{2} \frac{\partial^{2} v_{0}}{\partial t^{2}} \\
v_{1}(x, t)= & -\frac{1}{16} e^{-2 t+4 x}-\frac{5}{576} e^{-3 t+6 x}+\frac{5}{16} e^{-3 t+2 x} x-\frac{5}{64} e^{-3 t+2 x}-\frac{1}{4096} e^{-4 t+8 x} \\
& +\frac{1}{128} e^{-4 t+4 x} x-\frac{1}{512} e^{-4 t+4 x}-\frac{1}{48} e^{-4 t} x^{4}-\frac{1}{48} e^{-4 t} x^{3}-\frac{1}{128} e^{-4 t} x^{2} . \\
& \ldots
\end{aligned}
$$

In this manner, the remaining components of the homotopy perturbation solution can be obtained. This term clearly indicate that the homotopy perturbation method does not obtain efficient result to exact solution. Figs. 5 and 6 show the exact and approximate solutions by SHPM.

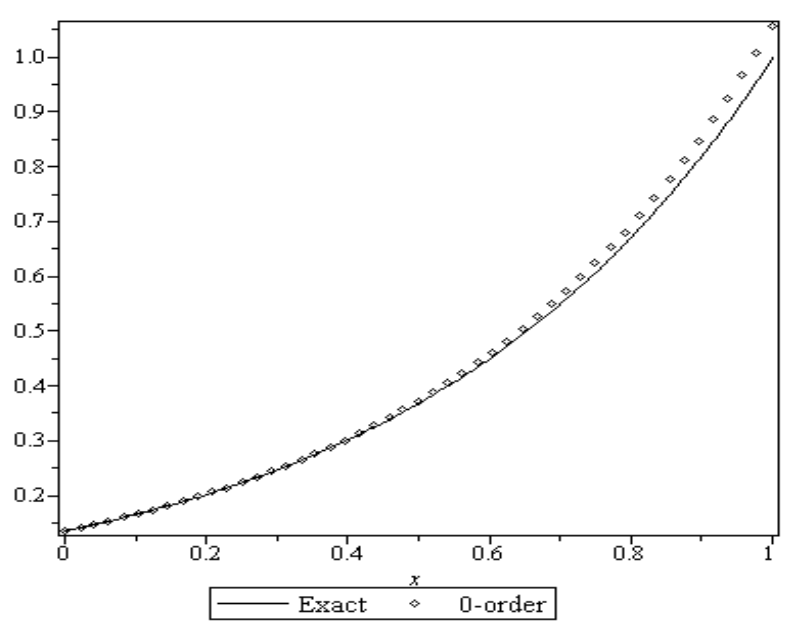

Fig. 5: Exact and 0-order of approximate solution by SHPM at $t=2$.

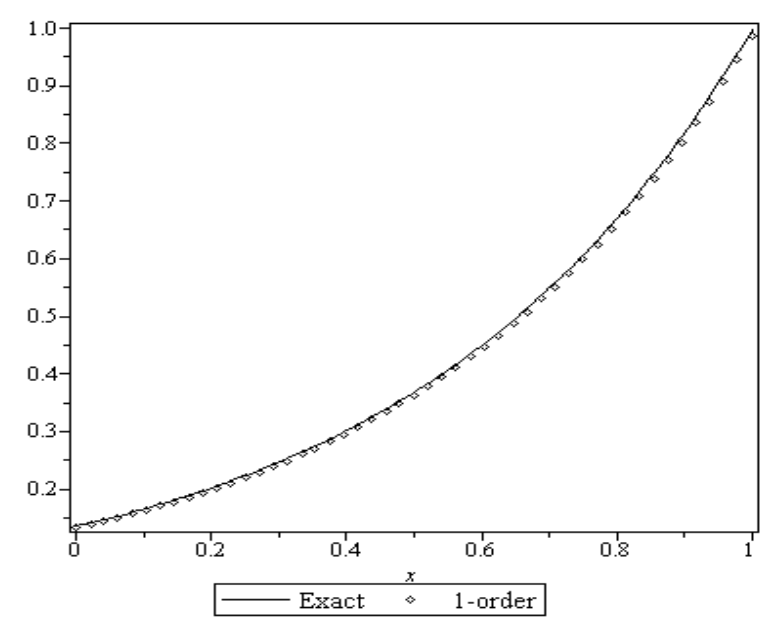

Fig. 6: Exact and 1-order of approximate solution by SHPM at $t=2$.

The modified HPM. In view of the homotopy (10), we construct the following homotopy 


$$
\frac{\partial^{2} u}{\partial x^{2}}-4 e^{-t+2 x}-p\left(-u \frac{\partial^{2} u}{\partial t^{2}}+e^{-2 t+4 x}\right)=0
$$

Substituting (6) into (19) and equating the terms with identical powers of $p$,we have

$$
\begin{aligned}
& p^{0}: \frac{\partial^{2} v_{0}}{\partial x^{2}}=4 e^{-t+2 x} \quad \square v_{0}(x, t)=e^{-t+2 x}, \\
& p^{1}: \frac{\partial^{2} v_{1}}{\partial x^{2}}=0 \quad \forall v_{1}(x, t)=0 . \ldots
\end{aligned}
$$

Consequently, solving the above equations, the first few components of the homotopy perturbation solution for Eq. (17)are derived as follows:

$$
v_{0}(x, t)=e^{-t+2 x}, \quad v_{k}=0, \quad k \geq 1
$$

The exact solution $u(x, t)=e^{-t+2 x}$ follows immediately.

Example 4. Consider the coupled system

$$
\begin{cases}\frac{\partial^{2} u}{\partial x^{2}}-\left(u \frac{\partial^{2} u}{\partial t^{2}}-v \frac{\partial^{2} v}{\partial t^{2}}\right)=1+\frac{3}{2}\left(x^{2}+t^{2}\right), & 0 \leq x, t \leq 1 / 2 \\ \frac{\partial^{2} v}{\partial x^{2}}-\left(u \frac{\partial^{2} v}{\partial t^{2}}+v \frac{\partial^{2} u}{\partial t^{2}}\right)=2\left(1-x^{2}-t^{2}\right), & \\ u(0, t)=\frac{t^{2}}{2}, \quad \frac{\partial}{\partial x} u(0, t)=0 ; \quad v(0, t)=t^{2}, & \frac{\partial}{\partial x} v(0, t)=0\end{cases}
$$

The exact solution of this system is

$$
u(x, t)=\frac{x^{2}+t^{2}}{2}, \quad v(x, t)=x^{2}+t^{2} .
$$

The standard HPM. We construct the following homotopy:

$$
\left\{\begin{array}{l}
H_{1}(u, v, p)=u(x, t)-h_{1}(x, t)-p \int_{0}^{x} \int_{0}^{x}\left(u \frac{\partial^{2} u}{\partial t^{2}}-v \frac{\partial^{2} v}{\partial t^{2}}\right) d x d x=0, \\
H_{2}(u, v, p)=v(x, t)-h_{2}(x, t)-p \int_{0}^{x} \int_{0}^{x}\left(u \frac{\partial^{2} v}{\partial t^{2}}+v \frac{\partial^{2} u}{\partial t^{2}}\right) d x d x=0
\end{array}\right.
$$

where

$$
\begin{gathered}
h_{1}(x, t)=\frac{t^{2}}{2}+\int_{0}^{x} \int_{0}^{x}\left(1+\frac{3}{2}\left(x^{2}+t^{2}\right)\right) d x d x \\
h_{2}(x, t)=t^{2}+\int_{0}^{x} \int_{0}^{x} 2\left(1-x^{2}-t^{2}\right) d x d x .
\end{gathered}
$$

Substituting (6) into (21) and equating the terms with identical powers of $p$, we have 


$$
\begin{aligned}
p^{0}: \quad u_{0}(x, t) & =h_{1}(x, t), \\
v_{0}(x, t) & =h_{2}(x, t) \\
p^{1}: \quad u_{1}(x, t) & =\int_{0}^{x} \int_{0}^{x}\left(u_{0} \frac{\partial^{2} u_{0}}{\partial t^{2}}-v_{0} \frac{\partial^{2} v_{0}}{\partial t^{2}}\right) d x d x \\
v_{1}(x, t) & =\int_{0}^{x} \int_{0}^{x}\left(u_{0} \frac{\partial^{2} v_{0}}{\partial t^{2}}+v_{0} \frac{\partial^{2} u_{0}}{\partial t^{2}}\right) d x d x \\
p^{2}: \quad u_{2}(x, t) & =\int_{0}^{x} \int_{0}^{x}\left(u_{0} \frac{\partial^{2} u_{1}}{\partial t^{2}}+u_{1} \frac{\partial^{2} u_{0}}{\partial t^{2}}-v_{0} \frac{\partial^{2} v_{1}}{\partial t^{2}}-v_{1} \frac{\partial^{2} v_{0}}{\partial t^{2}}\right) d x d x \\
v_{2}(x, t) & =\int_{0}^{x} \int_{0}^{x}\left(u_{0} \frac{\partial^{2} v_{1}}{\partial t^{2}}+u_{1} \frac{\partial^{2} v_{0}}{\partial t^{2}}+v_{0} \frac{\partial^{2} u_{1}}{\partial t^{2}}+v_{1} \frac{\partial^{2} u_{0}}{\partial t^{2}}\right) d x d x .
\end{aligned}
$$

$\vdots$

With the aid of symbolic computation of Maple, we can obtain the solutions:

$$
\begin{aligned}
p^{0}: \quad u_{0}(x, t) & =\frac{1}{2} t^{2}+\frac{1}{8} x^{4}+\frac{1}{2} x^{2}+\frac{3}{4} x^{2} t^{2} \\
v_{0}(x, t) & =t^{2}-\frac{1}{6} x^{4}+x^{2}-x^{2} t^{2} \\
p^{1}: & u_{1}(x, t)=-\frac{1}{384} x^{8}+\frac{77}{720} x^{6}-\frac{7}{240} x^{6} t^{2}+\frac{11}{24} x^{4} t^{2}-\frac{1}{8} x^{4}-\frac{3}{4} x^{2} t^{2}, \\
v_{1}(x, t) & =-\frac{1}{112} x^{8}+\frac{7}{360} x^{6}-\frac{1}{10} x^{6} t^{2}+\frac{1}{12} x^{4} t^{2}+\frac{1}{6} x^{4}-x^{2} t^{2} .
\end{aligned}
$$

Continuing this process, we find the three-term approximation as

$$
\begin{gathered}
u \cong u_{a}=u_{0}+u_{1}+u_{2}, \quad v \cong v_{a}=v_{0}+v_{1}+v_{2} . \\
u(x, t) \cong-\frac{527}{91238400} x^{16}-\frac{7453}{518918400} x^{14}+\frac{1}{8} x^{4}+\frac{1}{2} t^{2}+\frac{49}{720} x^{6} t^{2} \\
-\frac{11}{24} x^{4} t^{2}+\frac{1}{2} x^{2}+\frac{3}{4} x^{2} t^{2}+\frac{13}{960} x^{8}-\frac{11}{60} x^{6}+\frac{13949}{26611200} x^{12} \\
+\frac{779}{362880} x^{10}+\frac{299}{100800} x^{10} t^{2}+\frac{533}{40320} x^{8} t^{2}-\frac{527}{7862400} x^{14} t^{2} \\
-\frac{4321}{39916800} x^{12} t^{2}, \\
v(x, t) \cong \frac{-3089579}{435891456000} x^{16}-\frac{202457}{5448643200} x^{14}+\frac{36492997}{133382785536000} x^{18} t^{2}-
\end{gathered}
$$




$$
\begin{gathered}
\frac{10715}{13948526592} x^{16} t^{2}-\frac{1}{6} x^{4}+t^{2}+\frac{527}{6379388928000} x^{24}+\frac{7}{30} x^{6} t^{2}-\frac{1}{12} x^{4} t^{2}+ \\
\frac{61947029}{4827186524160000} x^{20}-\frac{12257249}{266765571072000} x^{18}+\quad \frac{118733}{4732923340590008000} x^{22}+\frac{13}{280} x^{8}-\frac{1}{30} x^{6}+ \\
\frac{31}{21794572800} x^{22} t^{2}+c x^{20}+\frac{857}{7484400} x^{12}-\frac{1913}{907200} x^{10}+\frac{29}{56700} x^{10} t^{2}- \\
\frac{53}{4032} x^{8} t^{2}+x^{2}-x^{2} t^{2}-\frac{223291}{3632428800} x^{14} t^{2}-\frac{439}{1247400} x^{12} t^{2} .
\end{gathered}
$$

Figs. 7, 8, 9 and 10 show the exact and approximate solutions by SHPM.

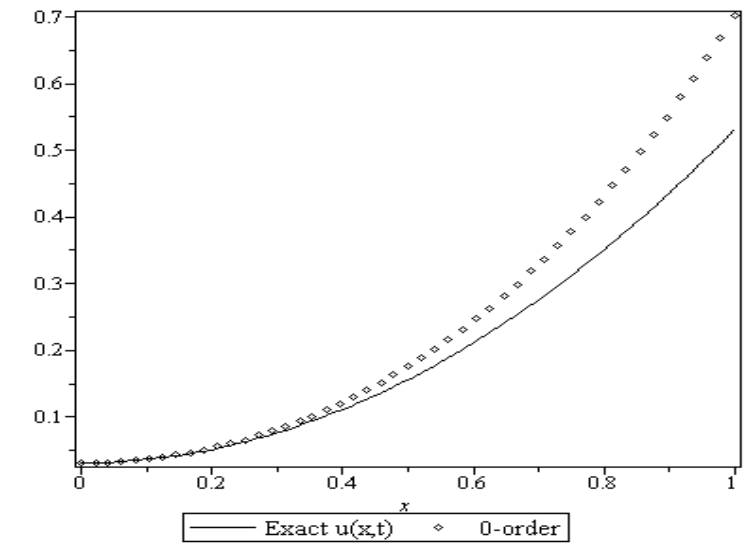

Fig. 7: Exact $u(x ; t)$ and one term of approximate solution by SHPM at $t=0: 25$.

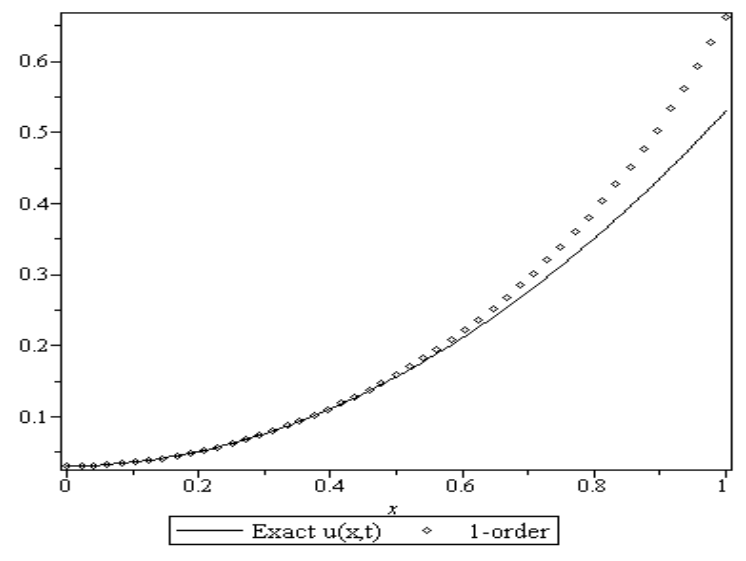

Fig. 8: Exact $u(x ; t)$ and two term of approximate solution by SHPM at $t=0: 25$.

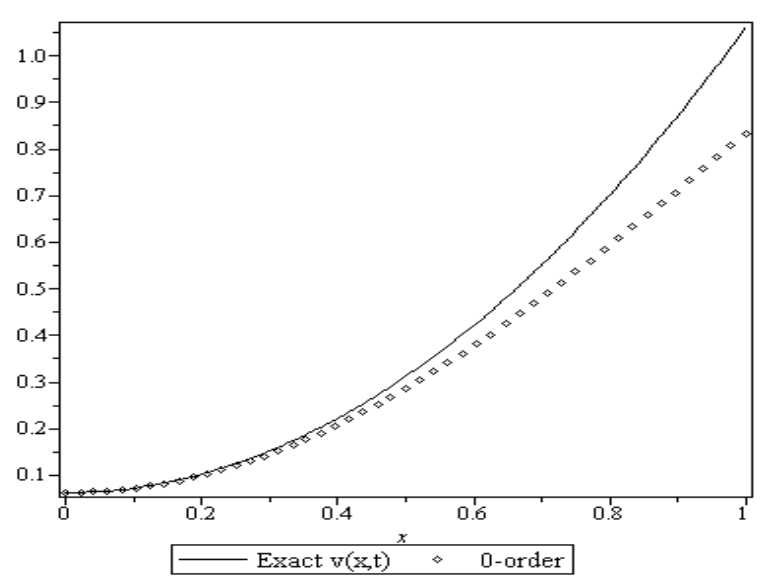

Fig. 9: Exact $v(x ; t)$ and one term of approximate solution $v 0$ by SHPM Fig.10: Exact $v(x ; t)$ and two term of approximate solution $v 0$ by at $t=0: 25$.

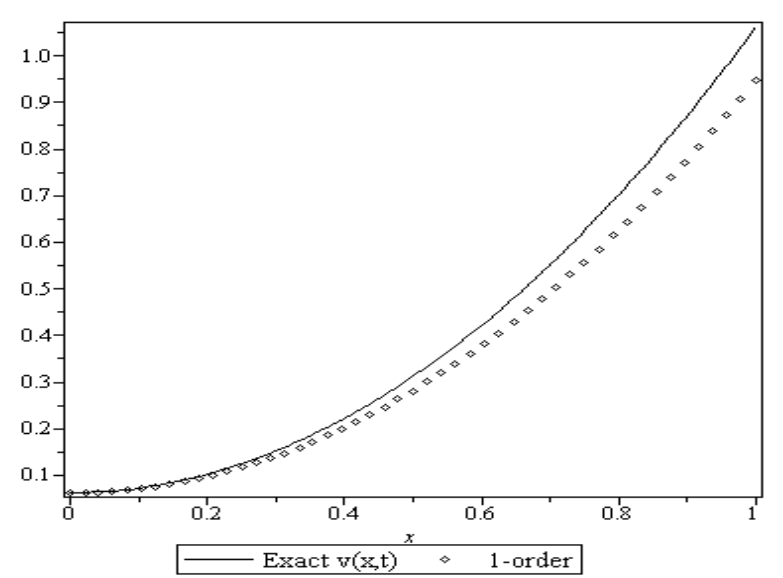

SHPM at $t=0: 25$.

The modified HPM. We construct the following homotopy 


$$
\left\{\begin{array}{l}
\frac{\partial^{2} u}{\partial x^{2}}-1-p\left(u \frac{\partial^{2} u}{\partial t^{2}}-v \frac{\partial^{2} v}{\partial t^{2}}+\frac{3}{2}\left(x^{2}+t^{2}\right)\right)=0 \\
\frac{\partial^{2} v}{\partial x^{2}}-2-p\left(u \frac{\partial^{2} v}{\partial t^{2}}+v \frac{\partial^{2} u}{\partial t^{2}}-2\left(x^{2}+t^{2}\right)\right)=0
\end{array}\right.
$$

Hence,

$$
\begin{aligned}
& p^{0}: \frac{\partial^{2} u_{0}}{\partial x^{2}}=1 \quad\left[u_{0}(x, t)=\frac{x^{2}+t^{2}}{2},\right. \\
& p^{0}: \frac{\partial^{2} v_{0}}{\partial x^{2}}=2 \quad \forall v_{0}(x, t)=x^{2}+t^{2}
\end{aligned}
$$

and

$$
u_{k}(x, t)=v_{k}(x, t)=0, \quad k=1,2, \cdots
$$

Therefore, the exact solution follows immediately.

\section{Conclusion}

In this paper, the modified HPM is applied to approximate solutions of non-linear wave and system of nonlinear wave equations. The results show us that this method can obtain the exact solution by only one iteration. It may be concluded that the modification method is remarkably effective and very simple for these kinds of problems.

\section{References}

[1] He J.H., Commun. Nonlinear Sci. Numer. Simul., 3, 92 (1998).

[2] Abbasbandy S., Appl. Math. Comput., 175, 581 (2006).

[3] Abbasbandy S., Chaos Solitons Fract., 31, 1243 (2007).

[4] He J.H., Chaos Soliton Fract., 26, 695 (2005).

[5] Ganji D.D., A. Sadighi, Int. J. Nonlinear Sci. Numer. Simul., 7, 411(2006).

[6] Jafari H., S. Seifi, Commun. Nonli. Science Numer. Simul., 14( 5) 2006 (2009).

[7] Jafari H., M. Zabihi and M. Saidy, Appl. Math. Sciences, 482393 (2008)

[8] Javidi M., A. Golbabai, Appl. Math. Comput., 189, 1921 (2007).

[9] Ramos J.I., Appl. Math. Comput., 198, 92 (2008).

[10] Wang Q., Appl. Math. Comput., 190, 1795 (2007).

[11] Odibat Z., Appl. Math. Comput., 189, 746 (2007).

[12] Ghasemi M., M. T. Kajani, A. Davari, Appl. Math. Comput.,189, 341 (2007). 
B. Ghazanfari, A.G. Ghazanfari, M. Fuladvand / TJMCS Vol .3 №.2 (2011) 212 - 224

[13] Roul P., Meyer P., Appl. Math. Model. 35, 4234 (2011).

[14] Singh J., Gupta P.K., Rai K.N., CIMS-DST Appl. Math. Model. 35, 1937 (2011).

[15] Gepreel K.A. Appl. Math. letters 24, 1428 (2011).

[16] Wazwaz A.M., J. Math. Anal. Appl., 5, 265 (1997).

[17] He J.H., Comput. Methods Appl. Mech. Eng., 167, 57 (1998). 